\title{
Arithmetic Properties of Overpartition Pairs into Odd Parts
}

\author{
Bernard L.S. Lin \\ School of Sciences \\ Jimei University, Xiamen, P.R. China \\ linlsjmu@163.com
}

Submitted: Jan 13, 2012; Accepted: Apr 22, 2012; Published: May 4, 2012

Mathematics Subject Classification: 05A17, 11P83

\begin{abstract}
In this work, we investigate various arithmetic properties of the function $\overline{p p}_{o}(n)$, the number of overpartition pairs of $n$ into odd parts. We obtain a number of Ramanujan type congruences modulo small powers of 2 for $\overline{p p}_{o}(n)$. For a fixed positive integer $k$, we further show that $\overline{p p}_{o}(n)$ is divisible by $2^{k}$ for almost all $n$. We also find several infinite families of congruences for $\overline{p p}_{o}(n)$ modulo 3 and two formulae for $\overline{p p}_{o}(6 n+3)$ and $\overline{p p}_{o}(12 n)$ modulo 3 .
\end{abstract}

Keywords: congruence, modular forms

\section{Introduction and statement of results}

An overpartition of the positive integer $n$ is a nonincreasing sequence of natural numbers whose sum is $n$ in which the first occurrence of a number may be overlined. Let $\bar{p}(n)$ denote the number of overpartitions of $n$. For convenience, we assume that there is only one overpartition of zero denoted by $\emptyset$. Properties of $\bar{p}(n)$ have been the subject of many recent studies $[5,6,8,9,11,13,14]$.

Recently, Hirschhorn and Sellers [10] studied the arithmetic properties of overpartitions using only odd parts. More recently, arithmetic properties of overpartition pairs have been considered by Bringmann and Lovejoy [3], Chen and the author [4], and Kim [12]. In this paper, we are concerned with the arithmetic properties of the number of overpartition pairs of $n$ into odd parts. An overpartition pair into odd parts is a pair of overpartitions $(\lambda, \mu)$ such that the parts of both overpartitions $\lambda$ and $\mu$ are restricted to be odd integers. For example, there are 8 overpartition pairs of 2 into odd parts:

$$
(1+1, \emptyset),(\overline{1}+1, \emptyset),(\overline{1}, 1),(\overline{1}, \overline{1}),(1,1),(1, \overline{1}),(\emptyset, 1+1),(\emptyset, \overline{1}+1) .
$$


Let $\overline{p p}_{o}(n)$ denote the number of overpartition pairs of $n$ into odd parts. Then the generating function for $\overline{p p}_{o}(n)$ is

$$
\overline{P P}_{o}(q)=\sum_{n=0}^{\infty} \overline{p p}_{o}(n) q^{n}=\frac{\left(-q ; q^{2}\right)_{\infty}^{2}}{\left(q ; q^{2}\right)_{\infty}^{2}}=\frac{\left(q^{2} ; q^{2}\right)_{\infty}^{6}}{(q ; q)_{\infty}^{4}\left(q^{4} ; q^{4}\right)_{\infty}^{2}} .
$$

Throughout this paper, we assume that $q$ is a complex number with $|q|<1$ and we adopt the following customary $q$-series notation:

$$
(a ; q)_{\infty}=\prod_{n=1}^{\infty}\left(1-a q^{n-1}\right) .
$$

In Section 2, we investigate arithmetic behavior of $\overline{p p}_{o}(n)$ modulo powers of 2 . In particular, we show the following two results.

Theorem 1.1. For any $n \geqslant 1$,

$$
\overline{p p}_{o}(n) \equiv \begin{cases}4(\bmod 8), & \text { if } n \text { is an odd square number } \\ 0(\bmod 8), & \text { otherwise. }\end{cases}
$$

Theorem 1.2. Assume the prime factorization of $n$ is given by

$$
n=2^{\alpha} \prod p_{i}^{u_{i}} \prod q_{j}^{v_{j}}
$$

where $p_{i} \equiv 1(\bmod 4)$ and $q_{j} \equiv 3(\bmod 4)$. Then $\overline{p p}_{o}(n) \equiv 0(\bmod 16)$ if and only if one of the following holds:

- $\alpha \geqslant 2$,

- $\alpha=1$ and at least one number among $u_{i}$ 's and $v_{j}$ 's is odd,

- $\alpha=0$ and at least one $v_{j}$ is odd,

- $\alpha=0$ and at least one $u_{i}$ is congruent to 3 modulo 4,

- $\alpha=0$ and at least two $u_{i}$ are congruent to 1 modulo 4;

At the end of Section 2, we prove the following theorem.

Theorem 1.3. Let $k$ be a positive integer. Then $\overline{p p}_{o}(n)$ is almost always divisible by $2^{k}$, namely,

$$
\lim _{X \rightarrow \infty} \frac{\sharp\left\{n \leqslant X: \overline{p p}_{o}(n) \equiv 0\left(\bmod 2^{k}\right)\right\}}{X}=1 .
$$

In Section 3, we aim to show divisibilities satisfied by $\overline{p p}_{o}(n)$ with modulus 3 .

Theorem 1.4. For $\alpha \geqslant 0$ and all $n \geqslant 0$,

$$
\begin{aligned}
\overline{p p}_{o}\left(9^{\alpha}(9 n+6)\right) & \equiv 0(\bmod 3), \\
\overline{p p}_{o}\left(9^{\alpha}(27 n+18)\right) & \equiv 0(\bmod 3) .
\end{aligned}
$$


Theorem 1.5. For all $n \geqslant 0$,

$$
\begin{aligned}
\overline{p p}_{o}(6 n+3) & \equiv(-1)^{n} \sigma(2 n+1)(\bmod 3), \\
\overline{p p}_{o}(12 n) & \equiv(-1)^{n+1}(\sigma(n)-\sigma(n / 4))(\bmod 3) .
\end{aligned}
$$

Here $\sigma(n)$ denotes the sum of positive divisors of $n$ and $\sigma(x)=0$ if $x \notin \mathbb{N}$.

\section{Congruences for $\overline{p p}_{o}(n)$ modulo powers of 2}

In this section, we want to establish congruences for $\overline{p p}_{o}(n)$ modulo small powers of 2 . We will require a number of properties of Ramanujan's functions $\varphi(q)$ and $\psi(q)$, namely,

$$
\begin{aligned}
& \varphi(q)=\sum_{n=-\infty}^{\infty} q^{n^{2}} \\
& \psi(q)=\sum_{n=0}^{\infty} q^{n(n+1) / 2}=\sum_{n=-\infty}^{\infty} q^{2 n^{2}-n} .
\end{aligned}
$$

The necessary properties of $\varphi(q)$ and $\psi(q)$ are given in the following lemmas.

\section{Lemma 2.1.}

$$
\begin{aligned}
& \varphi(q)=\left(-q ; q^{2}\right)_{\infty}^{2}\left(q^{2} ; q^{2}\right)_{\infty}, \\
& \psi(q)=\frac{\left(q^{2} ; q^{2}\right)_{\infty}^{2}}{(q ; q)_{\infty}}
\end{aligned}
$$

Proof. These two identities follow from Jacobi's triple product identity [1, p.35].

\section{Lemma 2.2.}

$$
\begin{aligned}
\varphi\left(-q^{2}\right)^{2} & =\varphi(q) \varphi(-q), \\
\varphi(q)^{2} & =\varphi\left(q^{2}\right)^{2}+4 q \psi\left(q^{4}\right)^{2} . \\
\psi(q)^{2} & =\varphi(q) \psi\left(q^{2}\right) .
\end{aligned}
$$

Proof. The first identity follows from (2.1). The last two identities can be proved by using series manipulations, see [1, pp. 40-41] for a proof.

To prove the congruences in this paper, we will frequently use the following congruence relations without explicitly mentioning it.

Lemma 2.3. For positive prime $p$, we have

$$
\begin{aligned}
(q ; q)_{\infty}^{p} & \equiv\left(q^{p} ; q^{p}\right)_{\infty}(\bmod p) \\
\varphi(-q)^{p} & \equiv \varphi\left(-q^{p}\right)(\bmod p)
\end{aligned}
$$


Proof. The first congruence identity follows from the following fact

$$
(1-q)^{p} \equiv 1-q^{p}(\bmod p) .
$$

The second congruence identity follows from the first congruence identity and the product representation for $\varphi(-q)$.

By the product formula (2.1) for $\varphi(q)$, we have

$$
\overline{P P}_{o}(q)=\sum_{n=0}^{\infty} \overline{p p}_{o}(n) q^{n}=\frac{\varphi(q)}{\varphi(-q)} .
$$

We shall begin by proving the following Ramanujan type identities, which are essential to congruences modulo small powers of two in this section.

\section{Theorem 2.1.}

$$
\begin{aligned}
\sum_{n=0}^{\infty} \overline{p p}_{o}(2 n) q^{n} & =\frac{\varphi(q)^{2}}{\varphi(-q)^{2}}, \\
\sum_{n=0}^{\infty} \overline{p p}_{o}(2 n+1) q^{n} & =4 \frac{\psi\left(q^{2}\right)^{2}}{\varphi(-q)^{2}} .
\end{aligned}
$$

Proof. By (2.3) and (2.6), we have

$$
\overline{P P}_{o}(q)=\frac{\varphi(q)^{2}}{\varphi(q) \varphi(-q)}=\frac{\varphi(q)^{2}}{\varphi\left(-q^{2}\right)^{2}} .
$$

Applying (2.4), we find that

$$
\overline{P P}_{o}(q)=\frac{\varphi\left(q^{2}\right)^{2}}{\varphi\left(-q^{2}\right)^{2}}+4 q \frac{\psi\left(q^{4}\right)^{2}}{\varphi\left(-q^{2}\right)^{2}}
$$

which is equivalent to identities (2.7) and (2.8).

Next, we wish to derive the generating function for $\overline{p p}_{o}(4 n+2)$ from $(2.7)$.

\section{Theorem 2.2.}

$$
\sum_{n=0}^{\infty} \overline{p p}_{o}(4 n+2) q^{n}=8 \frac{\left(q^{2} ; q^{2}\right)_{\infty}^{12}}{(q ; q)_{\infty}^{12}} .
$$

Proof. Applying (2.3) in (2.7), we obtain that

$$
\sum_{n=0}^{\infty} \overline{p p}_{o}(2 n) q^{n}=\frac{\varphi(q)^{4}}{\varphi\left(-q^{2}\right)^{4}} .
$$

Choosing the terms for which the power of $q$ is odd, we see that

$$
\sum_{n=0}^{\infty} \overline{p p}_{o}(4 n+2) q^{2 n+1}=\frac{\varphi(q)^{4}-\varphi(-q)^{4}}{2 \varphi\left(-q^{2}\right)^{4}} .
$$


By (2.4), we have

$$
\varphi(q)^{4}-\varphi(-q)^{4}=\left(\varphi(q)^{2}+\varphi(-q)^{2}\right)\left(\varphi(q)^{2}-\varphi(-q)^{2}\right)=16 q \varphi\left(q^{2}\right)^{2} \psi\left(q^{4}\right)^{2} .
$$

Combining these two identities together, we find that

$$
\sum_{n=0}^{\infty} \overline{p p}_{o}(4 n+2) q^{2 n+1}=8 q \frac{\varphi\left(q^{2}\right)^{2} \psi\left(q^{4}\right)^{2}}{\varphi\left(-q^{2}\right)^{4}} .
$$

Dividing both sides by $q$ and replacing $q^{2}$ by $q$, we get

$$
\sum_{n=0}^{\infty} \overline{p p}_{o}(4 n+2) q^{n}=8 \frac{\varphi(q)^{2} \psi\left(q^{2}\right)^{2}}{\varphi(-q)^{4}},
$$

which implies the desired result.

As an immediate consequence of the above theorem, we obtain the following congruences.

Corollary 2.1. For all $n \geqslant 0$,

$$
\begin{aligned}
\overline{p p}_{o}(12 n+6) & \equiv 0(\bmod 24), \\
\overline{p p}_{o}(12 n+10) & \equiv 0(\bmod 24) .
\end{aligned}
$$

We now want to prove the following theorem with the aid of Theorem 2.1.

Theorem 2.3. Let $d(n)$ denote the number of positive divisors of $n$. Then for all $n \geqslant 1$,

$$
\begin{aligned}
\overline{p p}_{o}(2 n) & \equiv 8(d(n)-d(n / 4))(\bmod 16), \\
\overline{p p}_{o}(2 n-1) & \equiv 4(-1)^{n-1} \sigma(2 n-1)(\bmod 32) .
\end{aligned}
$$

Here $d(x)=0$ if $x \notin \mathbb{N}$.

Proof. Using (2.5) in (2.8), we find that

$$
\sum_{n=0}^{\infty} \overline{p p}_{o}(2 n+1) q^{n}=4 \frac{\psi(-q)^{4}}{\varphi(-q)^{4}} .
$$

Now it is known that (see, e.g., Berndt [2, Chapter 3])

$$
\begin{aligned}
& \varphi(q)^{4}=1+8 \sum_{n=1}^{\infty} \frac{n q^{n}}{1+(-q)^{n}} \\
& \psi(q)^{4}=\sum_{n=0}^{\infty} \sigma(2 n+1) q^{n} .
\end{aligned}
$$


This gives

$$
\sum_{n=0}^{\infty} \overline{p p}_{o}(2 n+1) q^{n} \equiv 4 \sum_{n=0}^{\infty} \sigma(2 n+1)(-q)^{n}(\bmod 32) .
$$

Equating the coefficients $q^{n}$, we obtain (2.13). By (2.3), we see that

$$
\sum_{n=0}^{\infty} \overline{p p}(2 n) q^{n}=\frac{\varphi(-q)^{4} \varphi\left(-q^{2}\right)^{4}}{\varphi(-q)^{8}} .
$$

Applying (2.14) and the following congruence relation

$$
\varphi(-q)^{8} \equiv 1(\bmod 16)
$$

we get

$$
\begin{aligned}
\sum_{n=0}^{\infty} \overline{p p}(2 n) q^{n} & \equiv 1+8 \sum_{n=1}^{\infty} \frac{n(-q)^{n}}{1-(-q)^{n}}+8 \sum_{n=1}^{\infty} \frac{n\left(-q^{2}\right)^{n}}{1-\left(-q^{2}\right)^{n}}(\bmod 16) \\
& \equiv 1+8 \sum_{n=0}^{\infty} \frac{q^{2 n+1}}{1+q^{2 n+1}}+8 \sum_{n=0}^{\infty} \frac{q^{4 n+2}}{1+q^{4 n+2}}(\bmod 16) \\
& \equiv 1+8 \sum_{4 \nmid n} \frac{q^{n}}{1-q^{n}}(\bmod 16) \\
& \equiv 1+8 \sum_{n=1}^{\infty}(d(n)-d(n / 4)) q^{n}(\bmod 16) .
\end{aligned}
$$

This implies (2.12) and thus the proof is complete.

From Theorem 2.3, we have the following Ramanujan type congruences.

Corollary 2.2. For all $n \geqslant 0$,

$$
\begin{aligned}
& \overline{p p}_{o}(4 n+3) \equiv 0(\bmod 16), \\
& \overline{p p}_{o}(8 n+7) \equiv 0(\bmod 32) .
\end{aligned}
$$

Proof. Using the elementary fact that $\sigma(4 n+3)$ is divisible by 4 and $\sigma(8 n+7)$ is divisible by 8 , we immediately have the desired congruences.

With Theorem 2.3 in hand, we are now in a position to give a proof of Theorem 1.1. It is worth mentioning that Kim's [12] combinatorial argument for the characterization of the number of overpartition pairs of $n$ modulo 8 can also work for Theorem 1.1. Proof of Theorem 1.1. From (2.12) and (2.13), it follows that for any $n \geqslant 1$,

$$
\overline{p p}(2 n) \equiv 0(\bmod 8)
$$

and

$$
\overline{p p}(2 n-1) \equiv 4 \sigma(2 n-1)(\bmod 8) .
$$


Since $\sigma(2 n-1)$ is odd if and only if $2 n-1$ is a perfect square, we conclude that $\overline{p p}(2 n-1)$ is divisible by 8 if $2 n-1$ is not an odd perfect square and $\overline{p p}(2 n-1)$ is congruent to 4 modulo 8 if $2 n-1$ is an odd perfect square. This completes the proof.

By Theorem 2.3 and elementary properties of functions $d(n)$ and $\sigma(n)$, it is not hard to get Theorem 1.2, and we omit the details here. Theorem 1.2 produces infinite many congruences modulo 16 . We record two corollaries as follows.

Corollary 2.3. Let $p$ be an odd prime and let $r$ be an integer with $1 \leqslant r<p$. Then, for all $n \geqslant 0$,

$$
\overline{p p}_{o}(2 p(p n+r)) \equiv 0(\bmod 16) .
$$

Corollary 2.4. Let $p$ be a prime such that $p \equiv 1(\bmod 4)$ and let $r$ be an integer with $1 \leqslant r<p$. Then, for all $n \geqslant 0$,

$$
\overline{p p}_{o}\left(p^{3}(p n+r)\right) \equiv 0(\bmod 16) .
$$

To conclude this section, we shall show Theorem 1.3 by modular forms. It is worth mentioning that for any fixed positive integer $k$, Gordon and Ono [7] have proven that the number of partitions of $n$ into distinct parts is divisible by $2^{k}$ for almost all $n$, and Bringmann and Lovejoy [3] showed that the number of overpatition pairs of $n$ is also divisible by $2^{k}$ for almost all $n$.

Proof of Theorem 1.3. Recall that the Dedekind eta function $\eta(z)$ is defind by

$$
\eta(z)=q^{1 / 14} \prod_{n=1}^{\infty}\left(1-q^{n}\right)
$$

where $q=e^{2 \pi i z}$ and $z$ is in the upper plane of complex plane. Now we can rewrite $\overline{P P}_{o}\left(q^{24}\right)$ as the following eta quotient

$$
F(z)=\frac{\eta(48 z)^{6}}{\eta(24 z)^{4} \eta(96 z)^{2}} .
$$

Let

$$
f_{k}(z)=\frac{\eta(24 z)^{2^{k}}}{\eta(48 z)^{2^{k-1}}} .
$$

Observing that $f_{1}(z) \equiv 1(\bmod 2)$, it is not hard to establish the following fact by induction

$$
f_{k}(z) \equiv 1\left(\bmod 2^{k}\right)
$$

Define $G_{k}(z)$ by

$$
G_{k}(z)=F(z) f_{k}(z)=\frac{\eta(24 z)^{2^{k}-4}}{\eta(48 z)^{2^{k-1}-6} \eta(96 z)^{2}} .
$$

Thus,

$$
G_{k}(z) \equiv F(z)\left(\bmod 2^{k}\right)
$$


Without loss of generality, we may assume that $k \geqslant 3$. By [15, Thm 1.64 and Thm 1.65], it is not hard to check that $G_{k}(z)$ is a holomorphic modular form of weight $2^{k-2}$ on the congruence subgroup $\Gamma_{0}(1152)$. For the background on modular forms, see Ono [15]. From the deep theorem of Serre [15, p. 43], it follows that the Fourier coefficients of $G_{k}(z)$ is almost always divisible by $2^{k}$ and so are the Fourier coefficients of $F(z)$. Now

$$
F(z)=\sum_{n=0}^{\infty} \overline{p p}_{o}(n) q^{24 n}
$$

we see that almost all $n$ have the property that $\overline{p p}_{o}(n)$ is a multiple of $2^{k}$.

\section{Congruences for $\overline{p p}_{o}(n)$ modulo 3}

In this section, we aim to show Theorems 1.4 and 1.5. Before proving Theorem 1.4, we first give the following theorem.

Theorem 3.1. For all $n \geqslant 0$,

$$
\begin{gathered}
\overline{p p}_{o}(3 n) \equiv \overline{p p}_{o}(27 n)(\bmod 3), \\
\overline{p p}_{o}(9 n+6) \equiv 0(\bmod 3), \\
\overline{p p}_{o}(27 n+18) \equiv 0(\bmod 3) .
\end{gathered}
$$

The following 3-dissections of $\varphi(-q)$ and $\psi(q)$ are useful for the proof of Theorem 3.1.

\section{Lemma 3.1.}

$$
\begin{gathered}
\psi(q)=A\left(q^{3}\right)+q \psi\left(q^{9}\right), \\
\varphi(-q)=\varphi\left(-q^{9}\right)-2 q B\left(q^{3}\right),
\end{gathered}
$$

where

$$
A(q)=\frac{\left(q^{2} ; q^{2}\right)_{\infty}\left(q^{3} ; q^{3}\right)_{\infty}^{2}}{(q ; q)_{\infty}\left(q^{6} ; q^{6}\right)_{\infty}}, \quad B(q)=\frac{(q ; q)_{\infty}\left(q^{6} ; q^{6}\right)_{\infty}^{2}}{\left(q^{2} ; q^{2}\right)_{\infty}\left(q^{3} ; q^{3}\right)_{\infty}}
$$

Proof. Proofs of these two identities can be found in [1, p. 49].

Proof of Theorem 3.1. In this proof, all congruences hold to the modulus 3. By Lemma 2.3 , we see that

$$
\overline{P P}_{o}(q) \equiv \frac{\varphi(-q) \varphi\left(-q^{2}\right)^{2}}{\varphi\left(-q^{3}\right)} .
$$

Applying 3-dissection (3.5) of $\varphi(-q)$, we have

$$
\sum_{n=0}^{\infty} \overline{p p}_{o}(n) q^{n}=\frac{1}{\varphi\left(-q^{3}\right)}\left(\varphi\left(-q^{9}\right)-2 q B\left(q^{3}\right)\right)\left(\varphi\left(-q^{18}\right)-2 q^{2} B\left(q^{6}\right)\right)^{2}
$$


Choosing the terms for which the power of $q$ is a multiple of 3 , replacing $q^{3}$ by $q$, we obtain

$$
\sum_{n=0}^{\infty} \overline{p p}_{o}(3 n) q^{n} \equiv \frac{1}{\varphi(-q)}\left(\varphi\left(-q^{3}\right) \varphi\left(-q^{6}\right)^{2}-4 q B(q) B\left(q^{2}\right) \varphi\left(-q^{6}\right)\right) .
$$

By the the following identity due to Hirschhorn and Sellers [10, Lemma 3.4]

$$
\frac{\left(q^{3} ; q^{3}\right)_{\infty}^{3}}{(q ; q)_{\infty}}-4 q \frac{\left(q^{12} ; q^{12}\right)_{\infty}^{3}}{\left(q^{4} ; q^{4}\right)_{\infty}}=\frac{(q ; q)_{\infty}^{3}\left(q^{6} ; q^{6}\right)_{\infty}^{2}}{\left(q^{2} ; q^{2}\right)_{\infty}^{2}\left(q^{3} ; q^{3}\right)_{\infty}} \equiv\left(q^{2} ; q^{2}\right)_{\infty}\left(q^{6} ; q^{6}\right)_{\infty}
$$

we see that

$$
\begin{aligned}
\varphi\left(-q^{3}\right) \varphi\left(-q^{6}\right)-4 q B(q) B\left(q^{2}\right) & =\frac{\left(q^{3} ; q^{3}\right)_{\infty}^{2}\left(q^{6} ; q^{6}\right)_{\infty}}{\left(q^{12} ; q^{12}\right)_{\infty}}-4 q \frac{(q ; q)_{\infty}\left(q^{6} ; q^{6}\right)_{\infty}\left(q^{12} ; q^{12}\right)_{\infty}^{2}}{\left(q^{3} ; q^{3}\right)_{\infty}\left(q^{4} ; q^{4}\right)_{\infty}} \\
& =\frac{(q ; q)_{\infty}\left(q^{6} ; q^{6}\right)_{\infty}}{\left(q^{3} ; q^{3}\right)_{\infty}\left(q^{12} ; q^{12}\right)_{\infty}}\left(\frac{\left(q^{3} ; q^{3}\right)_{\infty}^{3}}{(q ; q)_{\infty}}-4 q \frac{\left(q^{12} ; q^{12}\right)_{\infty}^{3}}{\left(q^{4} ; q^{4}\right)_{\infty}}\right) \\
& \equiv \frac{(q ; q)_{\infty}\left(q^{2} ; q^{2}\right)_{\infty}\left(q^{6} ; q^{6}\right)_{\infty}^{2}}{\left(q^{3} ; q^{3}\right)_{\infty}\left(q^{12} ; q^{12}\right)_{\infty}}
\end{aligned}
$$

Thus,

$$
\begin{aligned}
\sum_{n=0}^{\infty} \overline{p p}_{o}(3 n) q^{n} & \equiv \frac{\varphi\left(-q^{6}\right)}{\varphi(-q)} \cdot \frac{(q ; q)_{\infty}\left(q^{2} ; q^{2}\right)_{\infty}\left(q^{6} ; q^{6}\right)_{\infty}^{2}}{\left(q^{3} ; q^{3}\right)_{\infty}\left(q^{12} ; q^{12}\right)_{\infty}} \\
& \equiv \frac{\left(q^{6} ; q^{6}\right)_{\infty}^{4}}{\left(q^{3} ; q^{3}\right)_{\infty}\left(q^{12} ; q^{12}\right)_{\infty}^{2}} \psi(q) \\
& =\frac{\left(q^{6} ; q^{6}\right)_{\infty}^{4}}{\left(q^{3} ; q^{3}\right)_{\infty}\left(q^{12} ; q^{12}\right)_{\infty}^{2}}\left(A\left(q^{3}\right)+q \psi\left(q^{9}\right)\right)
\end{aligned}
$$

where the last equality is obtained by the 3-dissection (3.4) of $\psi(q)$. From (3.7), we immediately deduce that for $n \geqslant 0$,

$$
\overline{p p}_{o}(9 n+6) \equiv 0
$$

and

$$
\begin{aligned}
\sum_{n=0}^{\infty} \overline{p p}_{o}(9 n) q^{n} & \equiv \frac{\left(q^{2} ; q^{2}\right)_{\infty}^{4}}{(q ; q)_{\infty}\left(q^{4} ; q^{4}\right)_{\infty}^{2}} A(q) \\
& \equiv \frac{\left(q^{3} ; q^{3}\right)_{\infty}\left(q^{6} ; q^{6}\right)_{\infty}}{\left(q^{12} ; q^{12}\right)_{\infty}}\left(q ; q^{2}\right)_{\infty}\left(q^{4} ; q^{4}\right)_{\infty} \\
& =\frac{\left(q^{3} ; q^{3}\right)_{\infty}\left(q^{6} ; q^{6}\right)_{\infty}}{\left(q^{12} ; q^{12}\right)_{\infty}} \sum_{n=-\infty}^{\infty}(-1)^{n} q^{2 n^{2}+n}
\end{aligned}
$$


Here the last equality is obtained by Jacobi's triple product identity. Because there are no integers $n$ such that $2 n^{2}+n$ congruent to 2 modulo 3 , we conclude that

$$
\overline{p p}_{o}(27 n+18) \equiv 0 \text {. }
$$

Since $2 n^{2}+n$ is divisible by 3 unless $n$ is congruent to 2 modulo 3 , we have

$$
\begin{aligned}
\sum_{\substack{n=-\infty \\
3 \mid 2 n^{2}+n}}^{\infty} q^{2 n^{2}+n} & =\sum_{n=-\infty}^{\infty} q^{2(3 n)^{2}+3 n}+\sum_{n=-\infty}^{\infty} q^{2(3 n+1)^{2}+3 n+1} \\
& =\sum_{n=-\infty}^{\infty}\left(q^{3}\right)^{n(3 n+1) / 2}
\end{aligned}
$$

From (3.9), we get

$$
\begin{aligned}
\sum_{n=0}^{\infty} \overline{p p}_{o}(27 n) q^{3 n} & \equiv \frac{\left(q^{3} ; q^{3}\right)_{\infty}\left(q^{6} ; q^{6}\right)_{\infty}}{\left(q^{12} ; q^{12}\right)_{\infty}} \sum_{\substack{n=-\infty \\
3 \mid 2 n^{2}+n}}^{\infty}(-1)^{n} q^{2 n^{2}+n} \\
& =\frac{\left(q^{3} ; q^{3}\right)_{\infty}\left(q^{6} ; q^{6}\right)_{\infty}}{\left(q^{12} ; q^{12}\right)_{\infty}} \sum_{n=-\infty}^{\infty}\left(-q^{3}\right)^{n(3 n+1) / 2}
\end{aligned}
$$

Therefore,

$$
\begin{aligned}
\sum_{n=0}^{\infty} \overline{p p}_{o}(27 n) q^{n} & \equiv \frac{(q ; q)_{\infty}\left(q^{2} ; q^{2}\right)_{\infty}}{\left(q^{4} ; q^{4}\right)_{\infty}} \sum_{n=-\infty}^{\infty}(-q)^{n(3 n+1) / 2} \\
& =\frac{(q ; q)_{\infty}\left(q^{2} ; q^{2}\right)_{\infty}}{\left(q^{4} ; q^{4}\right)_{\infty}} \cdot\left(q ;-q^{3}\right)_{\infty}\left(-q^{2} ;-q^{3}\right)_{\infty}\left(-q^{3} ;-q^{3}\right)_{\infty} \\
& =\frac{\left(q^{6} ; q^{6}\right)_{\infty}^{5}}{\left(q^{3} ; q^{3}\right)_{\infty}^{2}\left(q^{12} ; q^{12}\right)_{\infty}^{2}} \cdot \frac{(q ; q)_{\infty}^{2}}{\left(q^{2} ; q^{2}\right)_{\infty}}
\end{aligned}
$$

It is easy to check that

$$
\frac{\left(q^{6} ; q^{6}\right)_{\infty}^{4}}{\left(q^{3} ; q^{3}\right)_{\infty}\left(q^{12} ; q^{12}\right)_{\infty}^{2}} \cdot \frac{\left(q^{2} ; q^{2}\right)_{\infty}^{2}}{(q ; q)_{\infty}} \equiv \frac{\left(q^{6} ; q^{6}\right)_{\infty}^{5}}{\left(q^{3} ; q^{3}\right)_{\infty}^{2}\left(q^{12} ; q^{12}\right)_{\infty}^{2}} \cdot \frac{(q ; q)_{\infty}^{2}}{\left(q^{2} ; q^{2}\right)_{\infty}}(\bmod 3)
$$

from which we have

$$
\sum_{n=0}^{\infty} \overline{p p}_{o}(3 n) q^{n} \equiv \sum_{n=0}^{\infty} \overline{p p}_{o}(27 n) q^{n}(\bmod 3) .
$$

The desired result now follows by equating coefficients of $q^{n}, n \geqslant 0$, on both sides above. 
With Theorem 3.1 in hand, Theorem 1.4 follows immediately by induction on $\alpha$. Finally, we turn to show Theorem 1.5.

Proof of Theorem 1.5. From (3.6), we see that

$$
\sum_{n=0}^{\infty} \overline{p p}_{o}(3 n) q^{n} \equiv \varphi(q)^{2} \varphi\left(-q^{2}\right)^{2}(\bmod 3) .
$$

By the 2-dissection $(2.3)$ of $\varphi(q)^{2}$, it is not hard to get that

$$
\varphi(q)^{2} \varphi\left(-q^{2}\right)^{2}=\varphi\left(-q^{4}\right)^{4}+4 q \psi\left(-q^{2}\right)^{4} .
$$

It immediately follows that

$$
\begin{aligned}
\sum_{n=0}^{\infty} \overline{p p}_{0}(6 n+3) q^{n} & \equiv \psi(-q)^{4}(\bmod 3) \\
\sum_{n=0}^{\infty} \overline{p p}_{o}(6 n) q^{n} & \equiv \varphi\left(-q^{2}\right)^{4}(\bmod 3) .
\end{aligned}
$$

By (2.15) and the following identity (see, e.g., Berndt [2, p. 59])

$$
\varphi(q)^{4}=1+8 \sum_{n=1}^{\infty}(\sigma(n)-4 \sigma(n / 4)) q^{n},
$$

we obtain the desired results.

As a consequence of Theorem 1.5, we have the following corollaries.

Corollary 3.1. Let $p$ be prime with $p \equiv 2(\bmod 3)$ and let $r$ be an integer with $1 \leqslant r<p$. Then, for all $s \geqslant 0, n \geqslant 0$,

$$
\overline{p p}_{o}\left(3 p^{2 s+1}(p n+r)\right) \equiv 0(\bmod 3) .
$$

Proof. Note that $\sigma\left(p^{2 s+1}\right)=\frac{p^{2 s+2}-1}{p-1}$ is divisible by 3 and $\sigma\left(p^{2 s+1}(p n+r)\right)=\sigma\left(p^{2 s+1}\right) \sigma(p n+$ $r)$, so the result follows.

Corollary 3.2. Let $p$ be prime with $p \equiv 1(\bmod 3)$ and let $r$ be an integer with $1 \leqslant r<p$. Then, for all $s \geqslant 0, n \geqslant 0$,

$$
\overline{p p}_{o}\left(3 p^{3 s+2}(p n+r)\right) \equiv 0(\bmod 3) .
$$

Proof. Since $\sigma\left(p^{3 s+2}\right)$ is divisible by 3 , it is not hard to obtain the result by using the fact that $\sigma\left(p^{3 s+2}(p n+r)\right)=\sigma\left(p^{3 s+2}\right) \sigma(p n+r)$.

Corollary 3.3. For all $n \geqslant 0$,

$$
(-1)^{n} \overline{p p}_{o}(24 n+12) \equiv \overline{p p}_{o}(6 n+3)(\bmod 3) .
$$


Proof. From (1.5), we see that

$$
\overline{p p}_{o}(24 n+12) \equiv \sigma(2 n+1)-\sigma\left(\frac{2 n+1}{4}\right) \equiv \sigma(2 n+1)(\bmod 3),
$$

and so

$$
(-1)^{n} \overline{p p}_{o}(24 n+12) \equiv(-1)^{n} \sigma(2 n+1) \equiv \overline{p p}_{o}(6 n+3)(\bmod 3) .
$$

This completes the proof.

Acknowledgments. The author would like to thank the referee for helpful suggestions. This work incorporates part of the author's PhD thesis written at Nankai University under the direction of Professor William Y.C. Chen to whom the author expresses his gratitude. This work was supported by the Scientific Research Foundation of Jimei University, China.

\section{References}

[1] B.C. Berndt, Ramanujan's Notebooks, Part III, Springer-Verlag, New York, 1991.

[2] B.C. Berndt, Number Theory in the Spirit of Ramanujan, Amer. Math. Soc., Providence, 2006.

[3] K. Bringmann and J. Lovejoy, Rank and congruences for overpartition pairs, Int. J. Number Theory, 4 (2008), 303-322.

[4] W.Y.C. Chen and B.L.S. Lin, Arithmetic properties of overpartition pairs, Acta Arith., 151 (2012), 263-277.

[5] S. Corteel and J. Lovejoy, Overpartitions, Trans. Amer. Math. Soc., 356 (2004), 1623-1635.

[6] J.-F. Fortin, P. Jacob and P. Mathieu, Jagged partitions, Ramanujan J., 10 (2005), 215-235.

[7] B. Gordon and K. Ono, Divisibility of certain partition functions by powers of primes, Ramanujan J., 1 (1997), 25-34.

[8] M.D. Hirschhorn and J.A. Sellers, Arithmetic relations for overpartitions, J. Combin. Math. Combin. Comp., 53 (2005), 65-73.

[9] M.D. Hirschhorn and J.A. Sellers, An infinite family of overpartition congruences modulo 12, Integers, 5 (2005), Article A20.

[10] M.D. Hirschhorn and J.A. Sellers, Arithmetic properties of overpartitions into odd parts, Annals of Combinatorics, 10 (2006), 353-367.

[11] B. Kim, A short note on the overpartition function, Discrete Math., 309 (2009), 2528-2532.

[12] B. Kim, Overpartition pairs modulo powers of 2, Discrete Math., 311 (2011), 835-840.

[13] J. Lovejoy and R. Osburn, Quadratic forms and four partition functions modulo 3, Integers, 11 (2011), Article A4.

[14] K. Mahlburg, The overpatition function modulo small powers of 2, Discrete Math., 286 (2004), 263-267.

[15] K. Ono, The web of modularity: arithmetic of the coefficients of modular forms and $q$-series, CBMS Regional Conference Series in Mathematics, 102, Amer. Math. Soc., Providence, RI, 2004. 